INSTITUT NATIONAL DE RECHERCHE EN INFORMATIQUE ET EN AUTOMATIQUE

\title{
Efficient Dense Matching for Textured Scenes Using Region Growing
}

\author{
Maxime Lhuillier
}

$\mathbf{N}^{\circ} 3382$

Mars 1998

THÈME 3 



\title{
RIN RIA
}

\section{Efficient Dense Matching for Textured Scenes Using Region Growing}

\author{
Maxime Lhuillier \\ Thème 3 - Interaction homme-machine, \\ images, données, connaissances \\ Projet Movi \\ Rapport de recherche $\mathrm{n}^{\circ} 3382$ - Mars $1998-22$ pages
}

\begin{abstract}
We describe a simple and efficient dense matching method based on region growing techniques, which can be applied to a wide range of globally textured images. Our method can deal with non-rigid scenes and large camera motions. First a few highly distinctive features like points or areas are extracted and matched. These initial matches are then used in a correlation-based region growing step which propagates the matches in textured and more ambiguous regions of the images. The implementation of the algorithm is also given and is demonstrated on both synthetic and real image pairs.
\end{abstract}

Key-words: Dense Matching, Region Growing, Correlation 


\section{Appariement dense d'images texturées par croissance de régions}

Résumé : Un algorithme simple et rapide d'appariement dense utilisant des techniques de croissances de régions est présenté. Il s'applique à une large classe d'images texturées: scènes non rigides et grands déplacements. L'algorithme se décompose en deux étapes. On extrait et apparie d'abord des primitives, points ou régions, se distinguant facilement des autres. Ces premiers appariements sont ensuites utilisés pour initialiser une étape de croissance de régions appariées. Cette propagation d'appariements dans les zones texturées permet de construire, par continuité, les zones difficiles à apparier à partir de zones voisines plus facilement appariables. On décrit l'implémentation de l'algorithme, puis des tests sur des images synthétiques et réelles sont proposés.

Mots-clé : Appariement dense, Croissance de régions, Corrélation 


\section{Introduction}

Many algorithms have been proposed for dense matching. One popular approach is based on correlation, however this kind of algorithm is generally limited to relatively small disparity, hence small camera motion. For stereo images [DA89], [Kos93] whose epipolar geometry is known a priori, the search space can be reduced to a $1 \mathrm{D}$ along epipolar lines. Images rectification is usually used to accelerate the dense matching process, but does not allow zooming in/out of the camera.

Another approach is optical flow [BFB94], which handles non-rigid scenes but is limited to smaller displacements. Differential techniques give accurate estimation of displacements for smooth images, but fail for textured images and at depth discontinuities. Area-based matching techniques are fast, but do not perform well for sub-pixel displacements or dilations.

Occlusions are one of the major sources for wrong matches. Most of the recent stereo and optical flow work consists of incremental improvements to existing methods, to increase speed, accuracy or reliability. Only a few authors directly treat large occlusion stereo [IB94]. Hierarchical methods seem to be necessary to treat large disparity range. However, coarseto-fine strategies might miss some texture details and fail at depth discontinuities.

Our algorithm uses mainly region growing techniques. Region growing is a classic approach for segmentation [HS85], [Mon87], [AB94], and finding shapes [Bra93]. It is also well adapted to semi-interactive image processing applications [AB94] (Interactive process are unavoidable for real applications, although we will only discuss fully automatic techniques here). In its simplest sense, region growing is the process of merging neighboring points (or collections of points) into larger regions based on homogeneity properties (cf. [HS85]).

Matching by region growing, or in other words dense matching propagation, is implicitly used in regularization (fill the gaps in disparity map from neighboring matches) and optical flow techniques [BFB94] (by iterative algorithms of global cost minimization). An explicit region growing method is introduced in the photogrammetry domain by [OC89] with the "Gotcha" (Gruen-Otto-Chau) ALSC (Adaptative Least Square Correlation) algorithm. It starts with approximate patch matches between two SPOT satellite images and refines them. Their recovered distortion parameters are used to predict approximate matches for new patches in the neighborhood of the first match. Then, these patch matches are refined and so on. Complements for building extraction are discussed in the same domain by [KM96]: a pyramidal algorithm to produce seed matches and extraction of linear elements to remove possible blunders are proposed. Like Iterated Closest Point (ICP) methods for registration of $3 \mathrm{D}$ shapes [BM92], dense matching based on region growing combines a best first searching strategy and propagation to neighborhood.

Our main assumption is that the scene is globally textured like many outdoor scenes. Non-rigid scenes and large displacements are allowed. Our computation time is fast without image rectification, therefore camera zooming in/out is allowed.

Our algorithm has two main steps. The first step extracts and matches a sparse set of highly distinctive features (unlike to [KM96]): seed points and seed areas. Seed points are points of interest and are matched by correlation. If the scene is rigid, a robust technique to

$\mathrm{RR} \quad \mathrm{n}^{\circ} 3382$ 
match points of interest through the recovery of the unknown epipolar geometry [ZDFL94] could be used. Seed areas complete these matches in the most uniform colored areas. We extract and match them by simultaneously matching and region growing in the most uniform colored areas of the images.

The second step uses these initial matches to seed a dense matching propagation, using a best first matching strategy. This extends the matches to include the textured areas of the image. If the scene is rigid, we can use the epipolar geometry obtained in the first step to constrain the propagation in the second step. Our pixel-to-pixels propagation deals with fine texture details, and stops just at the occlusion borders if they are enough textured. The result is a pixelic matching, but it needs less calculations than patch-to-patches propagation and distortion parameter estimations like [OC89].

The report is organized as follows. The two steps of the dense matching algorithm are respectively described in Sections 2 and 3. Quantitative results on synthetic distortions and qualitative results on real image pairs are presented in Section 4. Section 5 summarizes the work, discusses its advantages and limitations and suggests some future directions.

\section{Initial Matching}

In this section, we show how to produce a set of initial candidate matches. We first justify the choice of seed points and seed areas. Secondly, we explain how to compare seed areas, and finally describe their matching and their region growing-based extraction.

\subsection{Which features to choose ?}

Matching points of interest is now a robust process, as demonstrated for example in [ZDFL94]. First, these points are extracted and matched with correlation. Because of noise and nearly repetitive patterns, a relaxation step and next a robust estimate of epipolar geometry seem to be necessary to produce reliable matches. However, a set of candidate point matches obtained by simple correlation could be sufficient to seed concurrent propagations.

Matching edge segments is well adapted for polyedric and low textured scenes. It is difficult to extract and match salient matches of edge segments in our case, because of our assumption of textured scenes.

Finally, it is known that segmentation is an instable process. Nevertheless, we need only to extract some initial seed area matches. A process will be described above which produces some reliable matches of the most uniform colored areas of the images. The simultaneous use of shape and mean color comparisons between some isolated and uniform colored regions in the globally textured images is sufficient to produce candidate seed area matches.

We use then seed areas and seed points. Such seed features could only be matched in weak distortion areas between the two images. Dense matching propagation will extend matching to more difficult and distorted regions to match. If the scene is rigid, the epipolar geometry is recovered while matching seed points. 


\subsection{How to Compare Two Seed Areas ?}

First, seed areas will not usually be distinguishable if their area are too small (unless their colors are rare or their neighborhoods are very different, but this case is not considered here). On the other hand, areas which are too large are subject to significant perspective and segmentation distortions. So we limit the minimum and maximum sizes of our seed areas. In practice, it turns out that the same interval of allowed values is sufficient for many different types of images. The range interval is 100 to 2000 pixels for all our tests.

Two areas $A$ and $B$ are compared very simply by their mean color and their shape (see Figure 1):
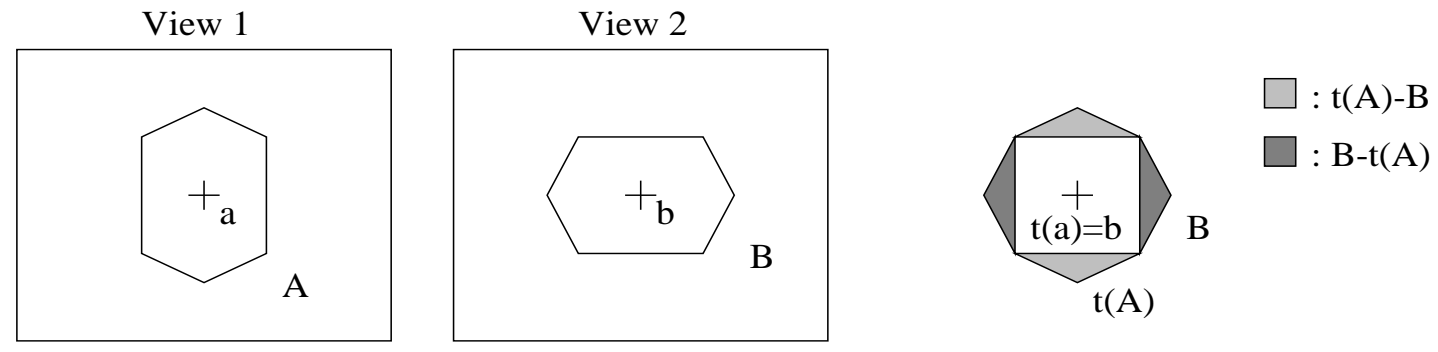

We want to compare areas A of view 1 and area B of view 2. Let a (resp. b) be the centroid of A (resp. B). Our simple shape-based criterion is the quotient of the gray surfaces by A's surface plus B's.

Figure 1: Comparison of two shapes.

$$
c(A, B)=\frac{|t(A)-B|+|B-t(A)|}{|A|+|B|}
$$

where $|A|$ is the area of $A$, "-" indicates set difference, and $t$ is the translation from $A$ 's centroid to $B$ 's. Areas are easily discriminated by their colors and forms, and $c$ allows for a little perspective distortion or initial segmentation error. Other measures could be used, such as the generalized Hausdorff measure [HJ95] or moments [BS97], but the two simple measures above have proved adequate in our experiments.

\subsection{Extract Candidate Matches for Seed Areas}

The first stage of our algorithm is an alternate sequence of region growing and matching steps for seed areas.

At the beginning, each pixel forms a separate region. During a growing step, for each connected $2 \times 2$ pixels block of pixels in the images, their regions are merged if their color difference is less than a threshold (see examples in Figure 2). The threshold is the same for all blocks, but increases between growing steps.

$\mathrm{RR} \mathrm{n}^{\circ} 3382$ 
During a matching step, each region of the first image is compared to each region of the second using the above criteria: candidate matches are accepted if both of their areas are within the thresholds and their mean color and shape differences are small.
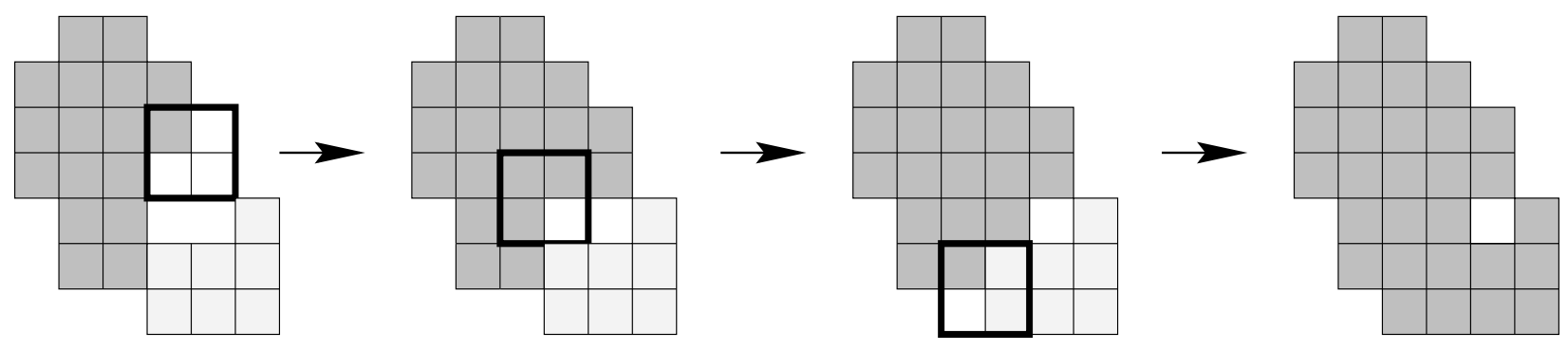

Each little square is a pixel. The big square is the selected $2 * 2$ block of pixel for a merge process. The difference between the max and min colors values of its pixel is bounded by the upper threshold mentioned in the text. The last merge is the fusion of two different region and a individual pixel.

Figure 2: Tree successive pixel or region merges using most uniform colored $2 \times 2$ pixels block.

The algorithm is run several times at different color uniformity levels for two reasons. Firstly, region growing is not strictly identical in the two images because of noise and perspective distortion: successive tests are necessary to ensure good matches. Secondly, it allows the same interval of tested thresholds to handle many different types of views.

\section{Dense Matching Propagation}

We have described the first step of our algorithm in the previous section. A method to obtain seed point matches was cited. An algorithm was also proposed to obtain seed area matches. The second step is now described. We first justify the choice of the dense matching propagation strategy; then we give the principle of the algorithm; finally the algorithm and some implementation details are given. For clarity, the exact link between the first and the second step is explicited in the last part of this section.

\subsection{Why Dense Matching Propagation ?}

Our goal is dense matching for textured, non differentiable and noisy images. We choose a correlation-based method because it is simple and fast. Correlation is less sensitive to geometric distortion if small windows are used. However, matching with small windows can be ambiguous with nearly periodic textures such as those of outdoor scenes. Thus, a strength constraint is needed for reliable matching. We use the continuity constraint: except for some pixels on the object boundaries, the disparity must vary smoothly. Dense matching 
propagation is a simple and effective way to use this constraint: the propagation moves from less ambiguous matches to more ambiguous ones.

\subsection{Principle}

A disparity map Map stores the region of correct pixel matches. The algorithm consists of growing this region. Let Start be a set of active pixel matches near its boundaries. At each step we remove the best match $m$ from set Start. Match $m$ is the seed for a local propagation: new matches in the neighborhood of $m$ (see Figure 3) are added simultaneously to set Start and map Map. New matches $(a, b)$ are added only if neither pixel $a$ nor $b$ are already matched in Map.

Neighborhood of pixel a in view 1 Neighborhood of pixel A in view 2
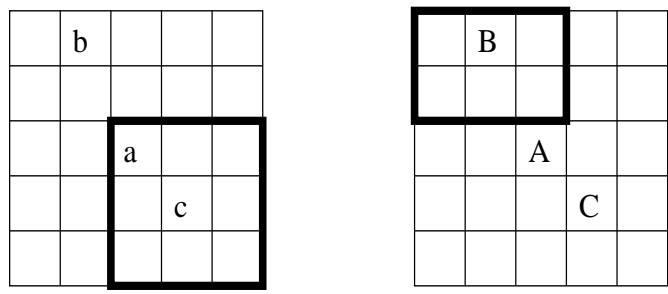

The neighborhood of a match $(\mathrm{a}, \mathrm{A})$ is a set of matchs included in the two 25-neighborhood of a and A. Possible correspondants of $b(\operatorname{resp} C)$ are in the black frame centered at B (resp c).

Figure 3: Definition of a match neighborhood.

Notice that:

- The set Start is always included in the region of correct matches in Map and initial content of Start.

- The unicity constraint is guaranteed in $M a p$ by our choice of new matches. Thus, the number of steps and size of set Start are bounded by the sum of the size of Start's initial content and the area of the image.

- Choosing only one match in the neighborhood of $m$ is inadequate. It does not produce a real $2 \mathrm{D}$ propagation, because size of set Start can not increase and so can not contain the whole boundaries of region in Map in progress.

- The risk of bad propagation is reduced by the choice of the best match $m$ of set Start.

Furthermore, the more textured the image, the lower the risk of bad propagation. We reduce the risk by forbidding local propagation in regions which are too smooth.

$\mathrm{RR} \mathrm{n}^{\circ} 3382$ 
Propagation is begun by initializing set Start as mentioned in Section 3.4. Propagation is stopped by image borders, too smooth regions and already matched areas. Occlusion contours stop it too, if they separate two different textures. If yes, they are included in borders of a finished propagation in one of the image. Difficult occlusions for others algorithms could so easily be localized with our algorithm.

\subsection{Implementation and Algorithm}

Disparity map Map is bidirectional and injective. We use a heap [Mon87] for the set Start to store the potential seeds for local propagations and to select the best at each step. The complexity of propagation is then $K n \log (n)$, where $n$ is the area of the image and $K$ a constant. Notice that it is independent of any disparity bound, and that $K$ is well known small. Let Local be a small auxiliary heap of pixel matches.

If $a$ is a pixel, let $\mathcal{N}_{x}(a)$ be the $x \times x$ window centered at pixel $a$. Let $s(a)$ be some estimate of the color roughness in $\mathcal{N}_{3}(a)$ and $s_{0}$ be a lower threshold. We use $s()$ to forbid propagation into insufficiently textured areas $\left\{a, s(a)<s_{0}\right\}$.

Let $d(a, b)$ be a measure of the image intensity/color difference between $\mathcal{N}_{3}(a)$ and $\mathcal{N}_{3}(b)$, and value $d_{0}$ be an upper threshold. The ratio $r(a, b)=\frac{\min (s(a), s(b))}{d(a, b)}$ is used as a measure of reliability for pixel match $(a, b)$. Matches with the best (the uppest) reliabilities are first considered.

Let $\left(r_{a}, g_{a}, b_{a}\right), 0 \leq r_{a}, g_{a}, b_{a}<1$ be the color of a pixel $a$. We use the following definitions:

$$
\begin{aligned}
n(a, b) & =.299\left|r_{a}-r_{b}\right|+.587\left|g_{a}-g_{b}\right|+.114\left|b_{a}-b_{b}\right| \\
d(a, b) & =\frac{1}{9} \sum_{\delta \in\{-1,0,1\} \times\{-1,0,1\}} n(a+\delta, b+\delta) \\
d_{0} & =0.07 \\
s(a) & =\max \{n(a, b), b-a \in\{(1,0),(-1,0),(0,1),(0,-1)\}\} \\
s_{0} & =0.04 \\
r(a, b) & =\frac{\min \{s(a), s(b)\}}{d(a, b)}
\end{aligned}
$$


The algorithm is

// First, initialize Start as mentioned in the next paragraph.

//Next, propagate:

Local $\leftarrow \emptyset$

while Start $\neq \emptyset$ do

- pull from Start the match $(a, b)$ which maximizes reliability $r(a, b)$

- // Store in Local the potential matches of local propagation:

- for each $(c, d)$ in $\left\{(c, d), c \in \mathcal{N}_{5}(a), d \in \mathcal{N}_{5}(b),(d-b)-(c-a) \in\{-1,0,1\} \times\{-1,0,1\}\right\}$ do

. . if $s(c)>s_{0}$ and $s(d)>s_{0}$ and $d(c, d)<d_{0} / /$ and possibles others constraints

. . then store match $(c, d)$ in the heap Local

. . end if

. end do

- // Store in Start and Map consistent matches of Local:

- while Local $\neq \emptyset$ do

. . pull from Local the match $(c, d)$ which maximizes $r(c, d)$

. . if $c$ and $d$ are not already stored in the disparity map Map then

. . store match $(c, d)$ in the disparity map Map

. . store match $(c, d)$ in the heap Start

. . end if

. end do

end do

If the scene is rigid, we add the epipolar constraint for match $(c, d)$ in the line if $s(c)>s_{0}$ and $s(d)>s_{0}$ and $d(c, d)<d_{0}$.

\subsection{Link between the First and Second Step}

The two steps were described in the previous sections. The first step produces candidate matches of seed points and seed areas, which are accurate up to a few pixels. The second step needs new candidate pixel matches with pixel accuracy.

If a seed point match is accurate about some pixels, it is a good trick to convert it to a set of concurrent, candidate pixel matches of its neighborhood. Best matches will be first selected, and a single good one is sufficient to provoke an avalanche of correct matches in the second step.

Seed area match $(A, B)$ is converted to concurrent, candidate pixel matches in set Start with the simple process below, where $t_{A B}$ (resp. $t_{B A}$ ) is the translation vector which maps A's centroid to B's (resp. B's to A's).

for each pixel a of A's boundaries do

store candidate match $\left(a, t_{A B}(a)\right)$ in the set Start

end do

for each pixel b of B's boundaries do

$\mathrm{RR} \mathrm{n}^{\circ} 3382$ 
store candidate match $\left(t_{B A}(b), b\right)$ in the set Start end do

These candidate matches of boundary pixels have proved adequate in our experiments to start a matching avalanche effect.

We combine the two steps using two possible strategies.

- All candidate matches start simultaneously concurrent propagations.

- Propagations are done one by one, best initial match first. A homogeneous reliability measure for seed point and seed area matches could be defined by the score of a first and bounded propagation.

Results are presented for the first strategy.

\section{Experimental Results}

Quantitative results on synthetic distortions and qualitative results on real image pairs are now discussed. It should be stressed that the same parameter values introduced in the various steps of the algorithm were used for all of the tests.

\subsection{Visualizing Arbitrary Dense Matching with a Checker-Board}

Since we test on non stereo pairs, disparity can not always be interpreted as depth, and it might also be large. Depth maps or displacement fields are not well adapted to display the results. We designed a global way to visualize dense matches for arbitrary images as follows. Pixels of the first image are colored with a gray-black checker-board. For each matched pixel of this image, we color the corresponding pixel of the second image with the same color. This makes it easy to visualize the match of each square and its distortion. A best way for color displays consists to blend a red-blue checked board with the original images.

\subsection{Synthetic Distortions}

Portions of variable textures from real outdoor images are collected to create image $I_{0}$ (see Figure 4). A second image $I_{1}$ is obtained from $I_{0}$ by translating the previous texture portions. Matchings between $I_{1}$ and $f\left(I_{0}\right)$ are now evaluated for some synthetic distortions $f$. The background is changed to forbid boundaries of textures portions to help propagation.

\subsubsection{Measures}

We evaluate coverage and accuracy of the dense matching propagation. For each texture and distortion $f$ the following numbers are now evaluated: 
- $S$ is the maximum percentage of matching coverage surface defined by the lower threshold value $s_{0}$. Remember that propagation is only allowed for pixels $a$ of area $\left\{a, s_{0}<s(a)\right\}$.

- $C$ is the percentage of matching coverage surface obtained (we have $C<S$ ).

- $E 1$ (resp. $E 2$ and $E 3$ ) is the percentage of pixel matches, such that their matching error is less than 1 pixel (resp. 2 and 3 pixels).

The matching error of a pixel match $(a, b)$ is defined by

$$
\operatorname{err}(a, b)=\max \left(|b-f(a)|,\left|a-f^{-1}(b)\right|\right)
$$

where $|$.$| is the Euclidean norm of the plane.$

\subsubsection{Evaluations}

Propagation is initialized in each couple of texture portions by a single seed point match at their centers. Figure 6 (resp. 7) shows S, C, E1, E2, E3 values for each texture and for successive 5, 10, 20, 30 degree rotations (resp. 5, 10, 20,30\% reductions). Figures 8 and 9 show the resulting distortions.

First, our visual matching checker-board suggests that the majority of the matches are good. One seed match suffices in majority of cases to propagate matching in the whole texture portion. Owing to the small correlation windows used, the most textured portions are matched for large distortions.

The larger distortion is, the lower matching coverage $C$ is. Thus, the percentage of good matches is usually high. More than $90 \%$ of pixel matches have less than 1 pixel error for 5,10 degree rotations and 5, $10 \%$ reductions, about $90 \%$ of pixel matches have less than 2 pixels error for 20 degree rotation or $20 \%$ reduction.

Banana texture is too smooth for $s_{0}$ value and is never matched. Grass1 texture contains low and dense details. Then, Grass 1 texture is subject to bad propagation (see $30 \%$ reduction). However, about $90 \%$ of pixel matches have less than 1 pixel error for 5,10 degree rotations and 5, $10 \%$ reductions (with matching coverage $45 \leq C \leq 50$ ). Bough0 texture contains strong details. Then, bad propagations are limited (see 30 degree rotation and $30 \%$ reductions). Like some real image pairs, Bough0 texture shows matching periodic holes (see 5, 10 degree rotations and 5, $10 \%$ reductions) dues to image discretization and uniform distortions.

\subsubsection{Automatic Seeding}

The goal of this paragraph is to show that correlation of points of interest is sufficient to produce good seed point matches. The test pair has very large disparities: images $I_{1}$ is matched with a 10 degree rotation of $I_{0}$ (see Figure 5). Each texture portion forms an isolated region: a portion is matched only if it contains a good seed point match. The results for matched portions are similar to previous ones.

$\mathrm{RR} \mathrm{n}^{\circ} 3382$ 
Maxime Lhuillier
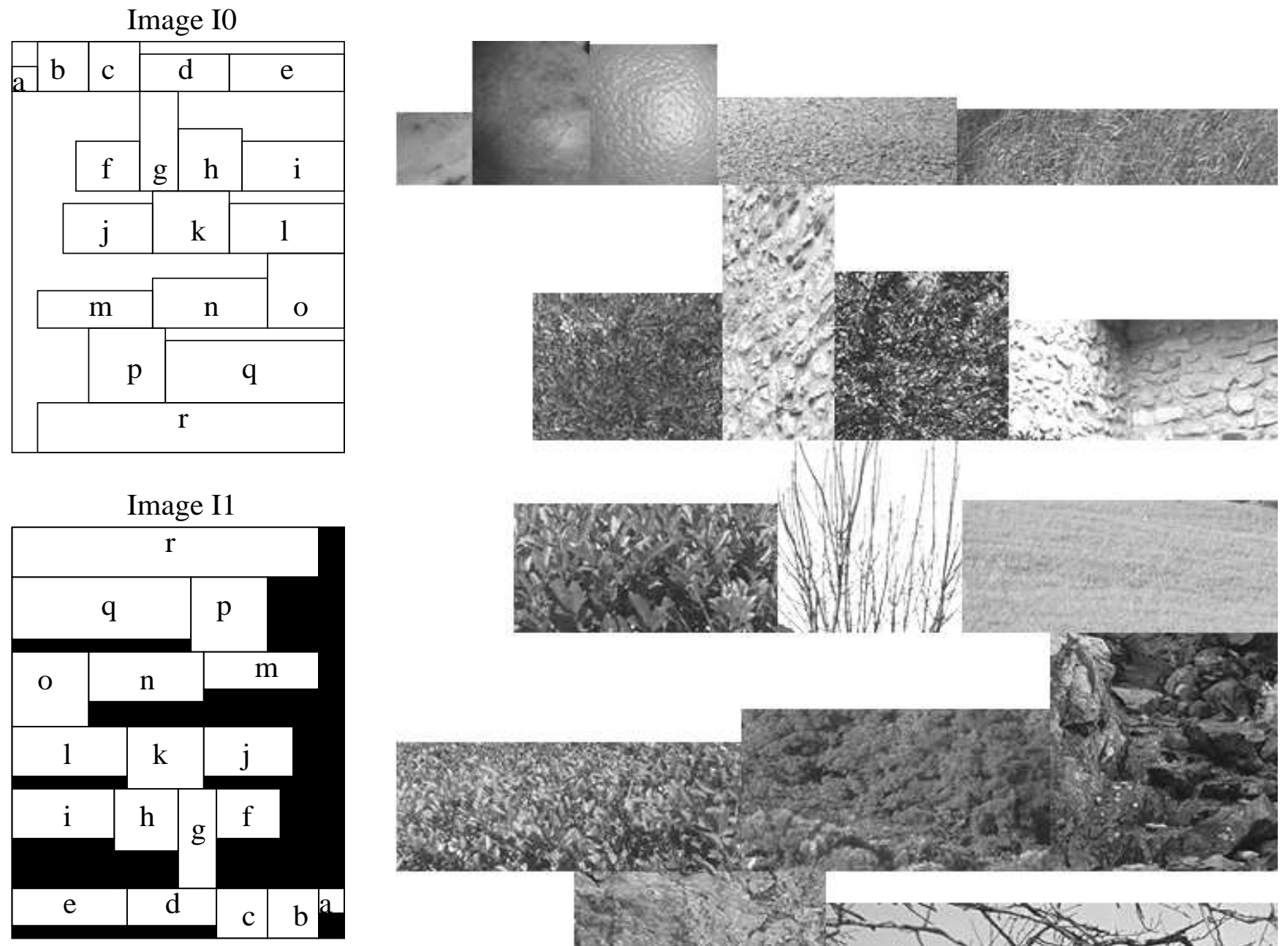

$\begin{array}{llll}\text { a } & \text { Banana } & \text { j } & \text { Foliage3 } \\ \text { b } & \text { Apple } & \text { k } & \text { Bough1 } \\ \text { c } & \text { Orange } & \text { l } & \text { Grass1 } \\ \text { d } & \text { Fine Gravel } & \text { m } & \text { Folliage2 } \\ \text { e } & \text { Grass0 } & \text { n } & \text { Folliage4 } \\ \text { f } & \text { Foliage1 } & \text { o } & \text { Rock1 } \\ \text { g } & \text { Wall0 } & \text { p } & \text { Rock0 } \\ \text { h } & \text { Foliage0 } & \text { q } & \text { Bough0 } \\ \text { i } & \text { Wall1 } & \text { r } & \text { Grass-Foliag }\end{array}$

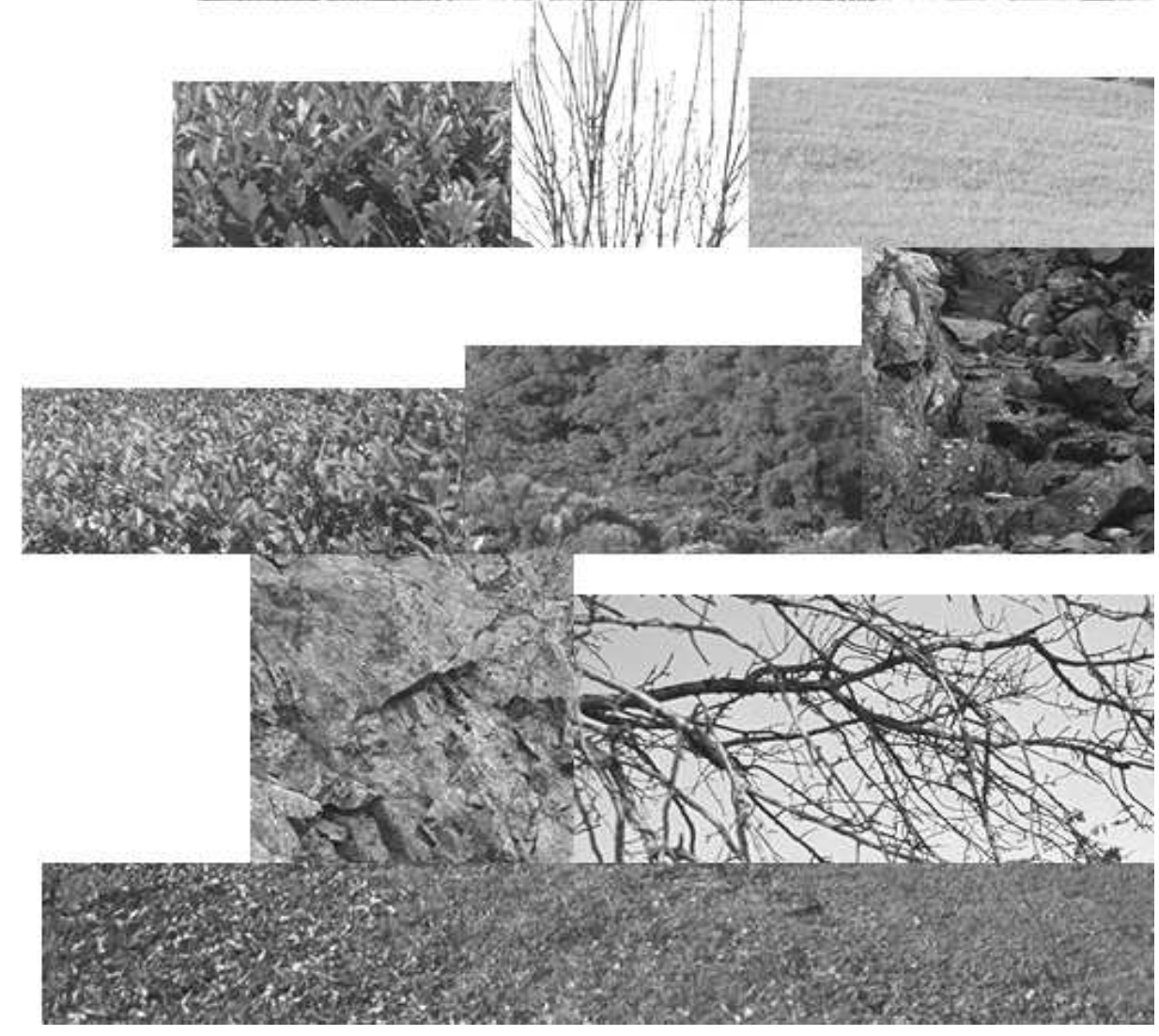

i Wall1 r Grass-Foliage

Figure 4: Textures for Tests. 


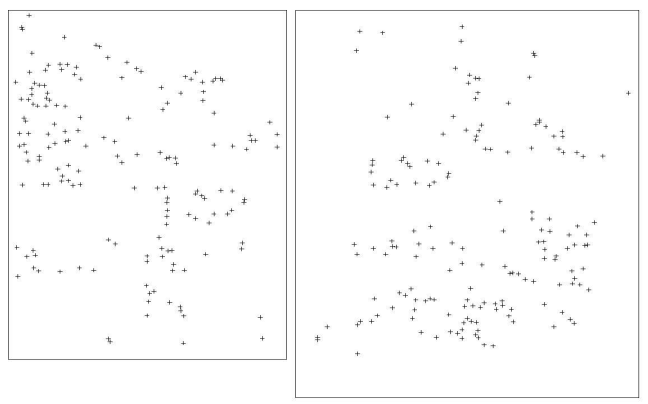

164 seed points matches (usually only one good seed point match per texture portion suffices).
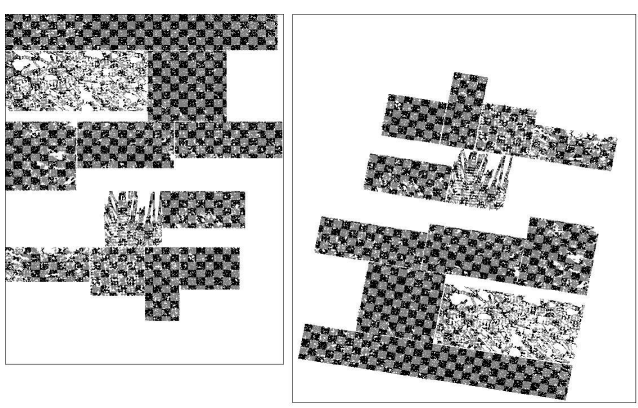

The propagation result : texture portions without good seed point matches are not matched.

Figure 5: Automatic seeding.

\subsection{Real image pairs}

The first scene is a textured rivulet (see Figure 11). Colored Image dimensions are $512 \times 768$. The following table summarizes the process and times on a Ultra SPARC 1, 300Mhz:

\begin{tabular}{|c|c|c|}
\hline Operations & Times & Results \\
\hline Detect Harris points and correlation & $5 \mathrm{~s}$ & 151 Seed point matches \\
\hline Extract and match most uniform regions & $14 \mathrm{~s}$ & 408 Seed area matches \\
\hline Dense Matching Propagation & $19 \mathrm{~s}$ & 238460 Pixel matches \\
\hline
\end{tabular}

The same detection and correlation as [ZDFL94] are used, but without relaxation or epipolar constraint. All seeds start concurrent propagation. Further, the dense matching propagation does not use epipolar constraint.

We show 6 images in Figure 11: the initial pair, the matched seed points and seed areas, and the dense matching propagation result. It should be noted that the initial pair is blurred on right down corner. Our visual "matching checker-board" suggests that the majority of the matches are good. Some seed matches are bad: the resulting propagations stop quickly thanks to good textures (see the small and isolated propagations near the border of the first image result).

Only a few seed matches are sufficient for highly textured scenes. We manually set 8 seed points matches and show propagation result in Figure 10. Manual correspondences are accurate to about 1-2 pixels. Only one seed match suffices to fill the majority of matching coverage (the background). However, more seed matches are necessary to obtain a similar result in the blurred region than the automatic seeding.

The second scene "street" is well textured too (cf. Figure 12). Results are obtained in the same conditions, and the majority of matches seems good without epipolar constraint. Tests on many others textured image pairs have also been successful. 


\begin{tabular}{|c||c|c|c|c|c||c|c|c|c|c||c|c|c|c|c||c|c|c|c|c||}
\hline & S & C & E1 & E2 & E3 & S & C & E1 & E2 & E3 & S & C & E1 & E2 & E3 & S & C & E1 & E2 & E3 \\
\hline Bough0 & 83 & 46 & 89 & 97 & 99 & 83 & 40 & 86 & 96 & 98 & 83 & 0 & 0 & 0 & 0 & 83 & 0 & 0 & 1 & 11 \\
\hline Grass0 & 98 & 84 & 97 & 99 & 99 & 98 & 82 & 96 & 99 & 99 & 98 & 79 & 85 & 96 & 99 & 98 & 73 & 52 & 69 & 78 \\
\hline Foliage0 & 99 & 70 & 98 & 99 & 99 & 99 & 65 & 97 & 99 & 99 & 99 & 51 & 90 & 97 & 99 & 99 & 35 & 67 & 84 & 92 \\
\hline Orange & 76 & 54 & 93 & 98 & 99 & 76 & 53 & 91 & 98 & 99 & 76 & 50 & 76 & 91 & 96 & 76 & 46 & 35 & 51 & 61 \\
\hline Apple & 35 & 6 & 91 & 97 & 98 & 35 & 0 & 53 & 69 & 84 & 35 & 0 & 50 & 75 & 87 & 35 & 0 & 71 & 71 & 71 \\
\hline Banana & 31 & 0 & 0 & 0 & 0 & 31 & 0 & 0 & 0 & 0 & 31 & 0 & 0 & 0 & 0 & 31 & 0 & 0 & 0 & 0 \\
\hline Grass-Foliage & 98 & 86 & 97 & 99 & 99 & 98 & 83 & 96 & 99 & 99 & 98 & 79 & 87 & 97 & 99 & 98 & 72 & 63 & 80 & 89 \\
\hline Wall0 & 97 & 81 & 96 & 99 & 99 & 97 & 80 & 95 & 99 & 99 & 97 & 73 & 87 & 97 & 99 & 97 & 66 & 67 & 87 & 94 \\
\hline Wall1 & 79 & 64 & 94 & 99 & 99 & 79 & 61 & 91 & 98 & 99 & 79 & 58 & 80 & 95 & 98 & 79 & 51 & 54 & 77 & 87 \\
\hline Fine Gravel & 99 & 86 & 97 & 99 & 99 & 99 & 84 & 95 & 97 & 98 & 99 & 81 & 86 & 95 & 98 & 99 & 73 & 54 & 69 & 79 \\
\hline Rock0 & 98 & 87 & 97 & 99 & 99 & 98 & 85 & 95 & 99 & 99 & 98 & 80 & 86 & 96 & 99 & 98 & 73 & 62 & 81 & 90 \\
\hline Rock1 & 91 & 78 & 95 & 99 & 99 & 91 & 76 & 93 & 99 & 99 & 91 & 72 & 83 & 96 & 99 & 91 & 66 & 58 & 80 & 90 \\
\hline Bough1 & 70 & 51 & 83 & 94 & 98 & 70 & 47 & 76 & 90 & 94 & 70 & 35 & 56 & 77 & 87 & 70 & 5 & 31 & 53 & 65 \\
\hline Foliage1 & 98 & 85 & 97 & 99 & 99 & 98 & 83 & 96 & 99 & 99 & 98 & 77 & 88 & 96 & 99 & 98 & 70 & 60 & 78 & 87 \\
\hline Foliage2 & 98 & 80 & 98 & 99 & 99 & 98 & 79 & 95 & 98 & 98 & 98 & 68 & 90 & 98 & 99 & 98 & 56 & 72 & 88 & 94 \\
\hline Foliage3 & 93 & 77 & 96 & 99 & 99 & 93 & 75 & 93 & 99 & 99 & 93 & 70 & 85 & 97 & 99 & 93 & 62 & 67 & 88 & 95 \\
\hline Grass1 & 81 & 50 & 94 & 98 & 99 & 81 & 48 & 92 & 98 & 99 & 81 & 44 & 75 & 87 & 93 & 81 & 35 & 16 & 24 & 29 \\
\hline Foliage4 & 94 & 82 & 96 & 99 & 99 & 94 & 81 & 93 & 98 & 99 & 94 & 79 & 84 & 96 & 99 & 94 & 76 & 62 & 82 & 92 \\
\hline
\end{tabular}

Figure 6: Matching coverages and accuracies for 5,10,20,30 degree rotations

\begin{tabular}{|c||c|c|c|c|c||c|c|c|c|c||c|c|c|c|c||c|c|c|c|c||}
\hline & S & C & E1 & E2 & E3 & S & C & E1 & E2 & E3 & S & C & E1 & E2 & E3 & S & C & E1 & E2 & E3 \\
\hline Bough0 & 83 & 45 & 88 & 97 & 99 & 83 & 40 & 82 & 94 & 98 & 83 & 17 & 67 & 86 & 93 & 83 & 0 & 47 & 67 & 83 \\
\hline Grass0 & 98 & 77 & 96 & 99 & 99 & 98 & 69 & 91 & 98 & 99 & 98 & 55 & 73 & 91 & 98 & 98 & 42 & 35 & 58 & 74 \\
\hline Foliage0 & 99 & 66 & 97 & 99 & 99 & 99 & 57 & 95 & 99 & 99 & 99 & 41 & 87 & 97 & 99 & 99 & 24 & 60 & 79 & 89 \\
\hline Orange & 76 & 52 & 93 & 99 & 99 & 76 & 46 & 87 & 97 & 99 & 76 & 40 & 56 & 79 & 90 & 76 & 30 & 7 & 16 & 23 \\
\hline Apple & 35 & 8 & 88 & 96 & 98 & 35 & 6 & 85 & 95 & 99 & 35 & 8 & 60 & 84 & 94 & 35 & 7 & 8 & 16 & 27 \\
\hline Banana & 31 & 1 & 0 & 0 & 0 & 31 & 0 & 0 & 0 & 0 & 31 & 0 & 0 & 0 & 0 & 31 & 0 & 0 & 0 & 0 \\
\hline Grass-Foliage & 98 & 79 & 96 & 99 & 99 & 98 & 72 & 94 & 99 & 99 & 98 & 56 & 78 & 94 & 99 & 98 & 42 & 49 & 71 & 82 \\
\hline Wall0 & 97 & 77 & 95 & 99 & 99 & 97 & 69 & 93 & 99 & 99 & 97 & 55 & 80 & 96 & 99 & 97 & 41 & 57 & 84 & 94 \\
\hline Wall1 & 79 & 61 & 92 & 98 & 99 & 79 & 55 & 87 & 97 & 99 & 79 & 46 & 68 & 89 & 96 & 79 & 35 & 38 & 60 & 74 \\
\hline Fine Gravel & 99 & 80 & 97 & 99 & 99 & 99 & 72 & 94 & 98 & 99 & 99 & 57 & 76 & 92 & 98 & 99 & 43 & 40 & 62 & 77 \\
\hline Rock0 & 98 & 81 & 96 & 99 & 99 & 98 & 73 & 92 & 98 & 99 & 98 & 58 & 77 & 95 & 99 & 98 & 44 & 49 & 76 & 89 \\
\hline Rock1 & 91 & 72 & 93 & 99 & 99 & 91 & 66 & 90 & 98 & 99 & 91 & 53 & 71 & 92 & 98 & 91 & 40 & 44 & 72 & 87 \\
\hline Bough1 & 70 & 50 & 76 & 89 & 95 & 70 & 45 & 72 & 89 & 96 & 70 & 32 & 50 & 69 & 79 & 70 & 10 & 13 & 23 & 31 \\
\hline Foliage1 & 98 & 79 & 96 & 99 & 99 & 98 & 71 & 95 & 99 & 99 & 98 & 55 & 80 & 95 & 99 & 98 & 41 & 49 & 72 & 85 \\
\hline Foliage2 & 98 & 76 & 96 & 99 & 99 & 98 & 68 & 95 & 99 & 99 & 98 & 53 & 85 & 96 & 98 & 98 & 36 & 68 & 90 & 96 \\
\hline Foliage3 & 93 & 73 & 94 & 99 & 99 & 93 & 66 & 91 & 98 & 99 & 93 & 53 & 77 & 95 & 99 & 93 & 39 & 53 & 81 & 92 \\
\hline Grass1 & 81 & 49 & 93 & 98 & 99 & 81 & 45 & 86 & 96 & 99 & 81 & 38 & 53 & 77 & 91 & 81 & 27 & 1 & 4 & 7 \\
\hline Foliage4 & 94 & 76 & 95 & 99 & 99 & 94 & 70 & 92 & 98 & 99 & 94 & 56 & 70 & 92 & 98 & 94 & 44 & 37 & 65 & 80 \\
\hline
\end{tabular}

Figure 7: Matching coverages and accuracies for 5,10,20,30 \% reductions

INRIA 

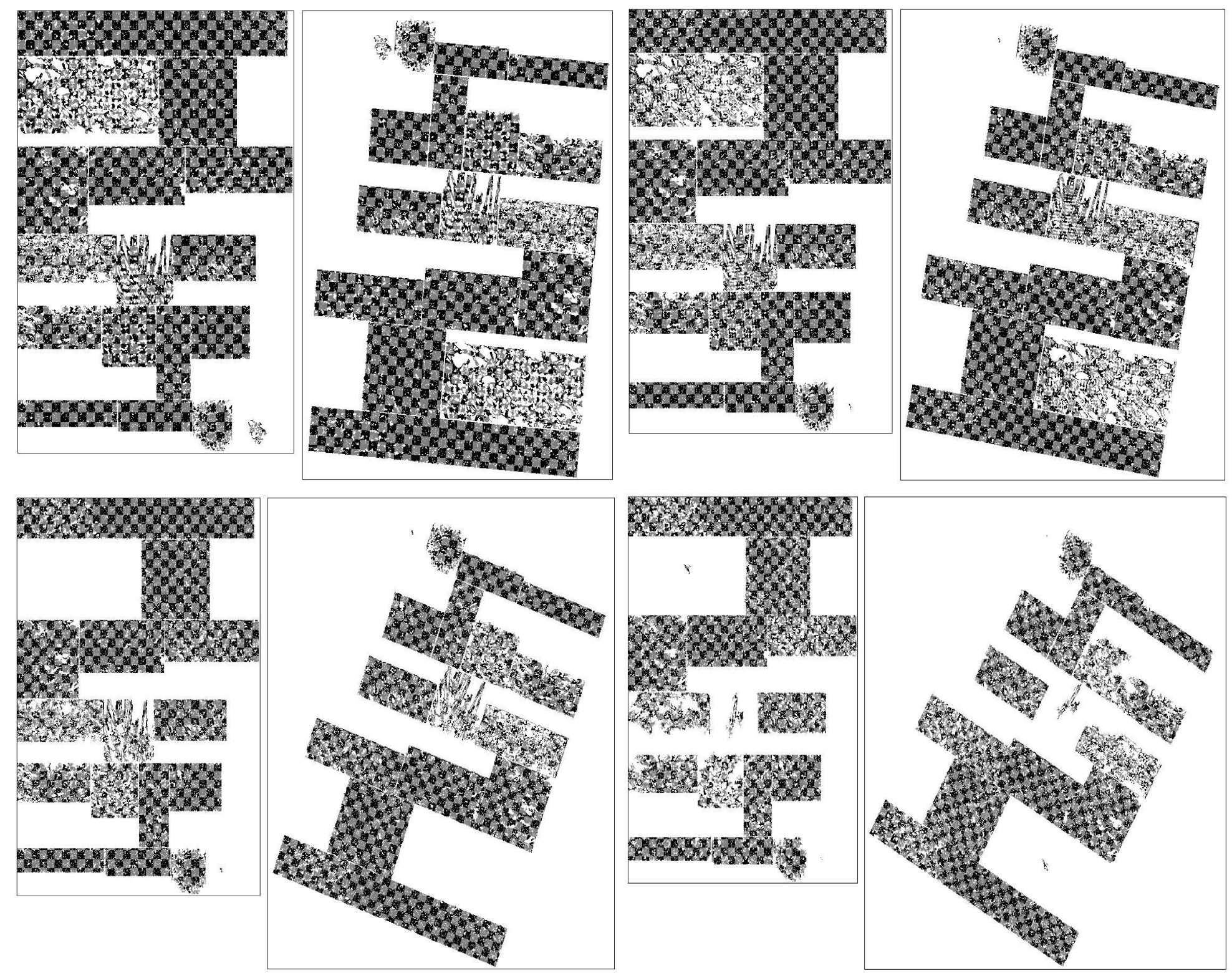

Figure 8: Matching for 5, 10, 20, 30, degree rotations. 
Maxime Lhuillier
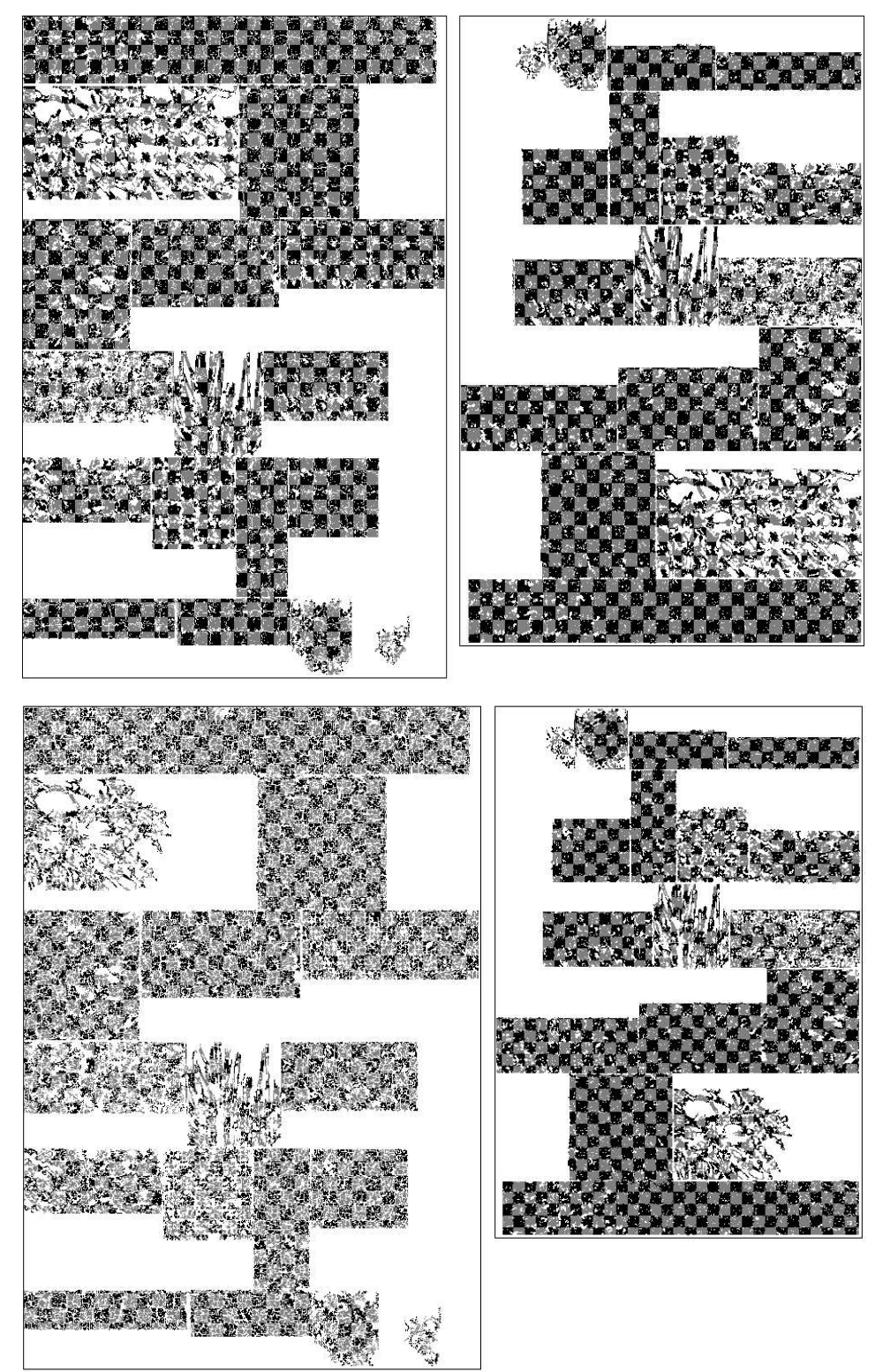
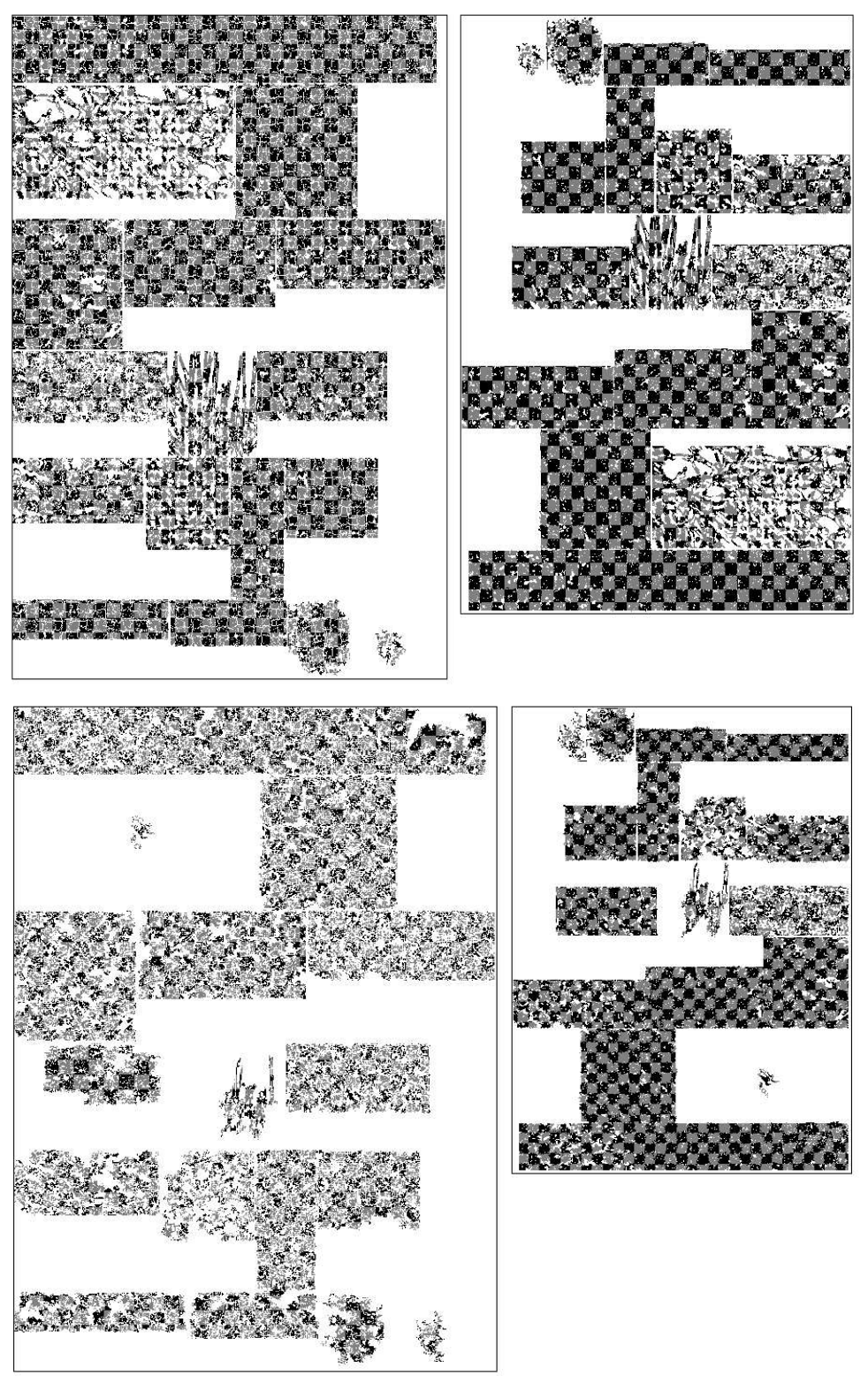

Figure 9: Matching for 5, 10, 20, $30 \%$ reductions. 

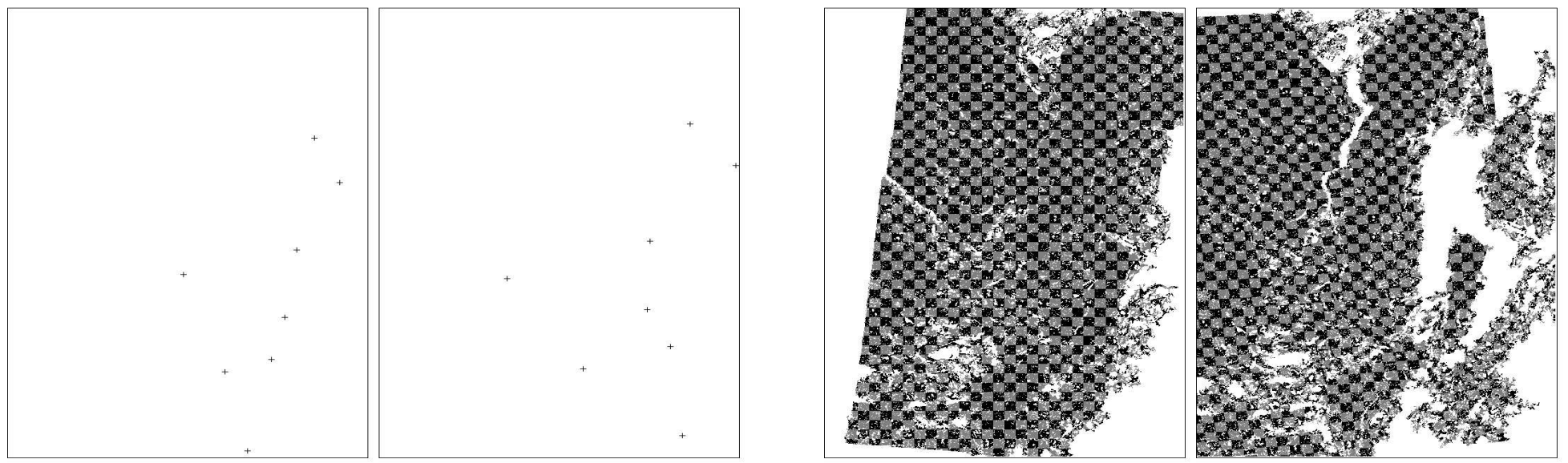

Figure 10: The result for "rivulet" image pair (some manual seed points).

We show finally the result for the less textured scene "bowl" (cf. Figure 13). Two propagations are then tested with lower roughness limits $\left(s_{0}=0.02\right.$ and $\left.s_{0}=0.01\right)$ to obtain more matches. Since less texture information is available, the recovered epipolar constraint is needed to limit bad propagations. Only contours and well textured areas are matched for $s_{0}=0.02$. Good results are obtained for the whole bowl for $s_{0}=0.01$. Indeed, propagation comes from textured borders of fruits to less textured areas inside. However, the majority of matches are bad for the table.

\section{Conclusion}

A new method has been proposed for dense matching two textured images. The algorithm has two main steps. First, we extract and match the most uniform colored areas and interest points. Then, a correlation-based match propagation is started from these seeds, to produce a dense matching covering all sufficiently textured areas of the images. We have successfully tested the algorithm on textured images with large displacements and distortions. Rigidity constraint is not indispensable for sufficiently textured scenes. However, our method is not suitable for non-textured images like indoor scenes and manufactured objects: the dense matching propagation is immediately stopped.

If an initially matched seed is false and its neighborhood was little textured, the resulting propagation may not stop quickly. Such cases could be detected by limiting the global deformation allowed between the two images. Other constraints could also be introduced to avoid it too, such as matching compactness. 

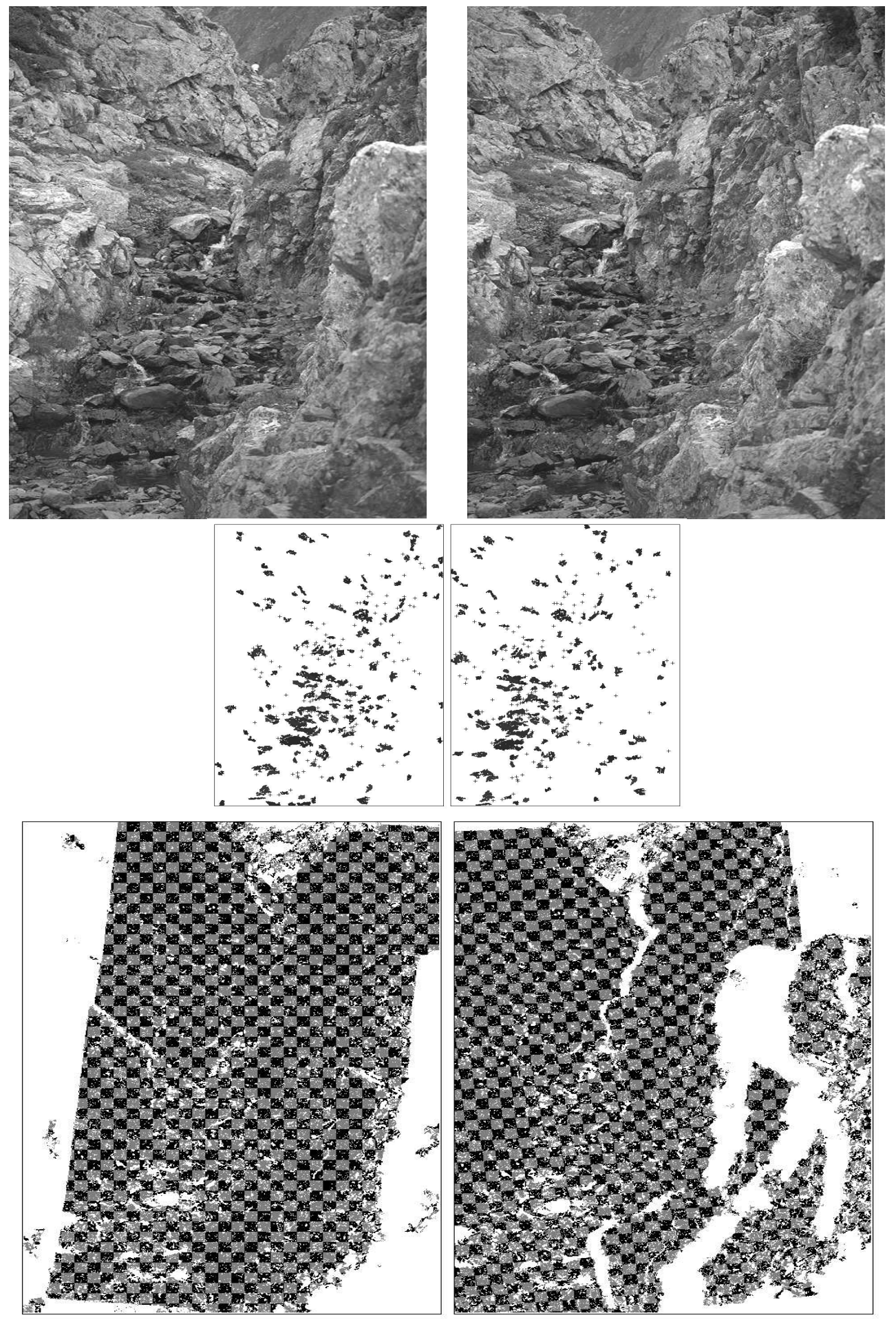

Figure 11: The result for "rivulet" image pair (automatic seeding).

INRIA 



Figure 12: The result for "street" image pair. 

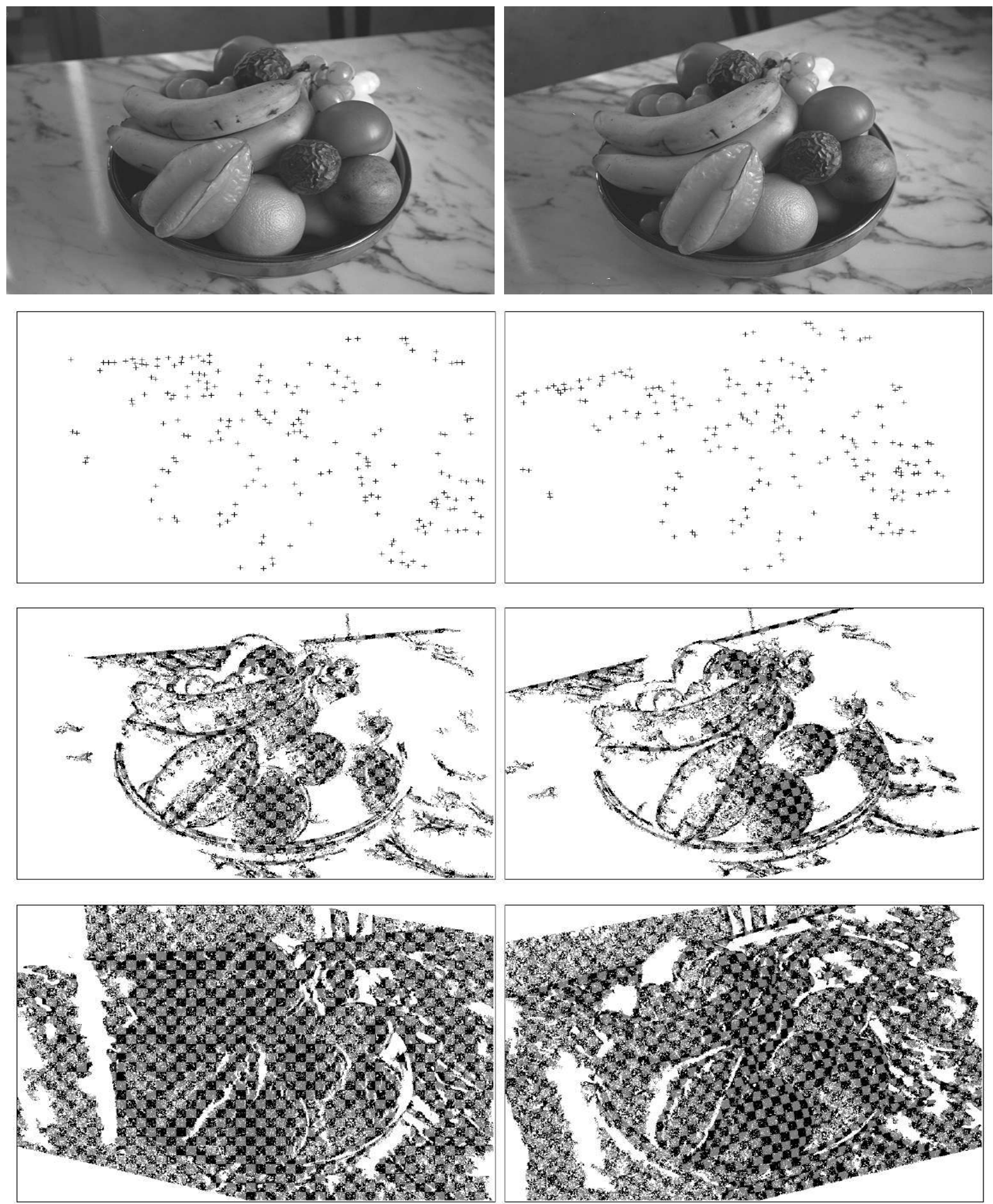

Figure 13: The result for "bowl" image pair.

INRIA 


\section{References}

[AB94] R. Adams and L. Bischof. Seeded region growing. IEEE Transactions on Pattern Analysis and Machine Intelligence, 16(6):641-647, June 1994.

[BFB94] J. Barron, D. Fleet, and S. Beauchemin. Performance of optical flow techniques. International Journal of Computer Vision, 12(1):43-77, 1994.

[BM92] P.J. Besl and N.D. McKay. A method for registration of 3D shapes. IEEE Transactions on Pattern Analysis and Machine Intelligence, 14(2):239-256, 1992.

[Bra93] M. Brand. A short note on local region growing by pseudophysical simulation. Proceedings of the Conference on Computer Vision and Pattern Recognition, New York, USA, pages 782-783, 1993.

[BS97] D. Bhattacharya and S. Sinha. Invariance of stereo images vie the theory of complex moments. Pattern Recognition, 30(9):1373-1387, 1997.

[DA89] U.R. Dhond and J.K. Aggarwal. Structure from stereo - a review. IEEE Transactions on Systems, Man and Cybernetics, 19(6):1489-1510, November 1989.

[HJ95] D.P. Huttenlocher and E.W. Jaquith. Computing visual correspondence: Incorporating the probability of a false match. In Proceedings of the 5th International Conference on Computer Vision, Cambridge, Massachusetts, USA, pages 515$522,1995$.

[HS85] R.M. Haralick and L.G. Shapiro. Image segmentation techniques. Computer Vision, Graphics, and Image Processing, 29:100-132, 1985.

[IB94] S.S. Intille and A.F. Bobick. Disparity-space images and large occlusion stereo. In Proceedings of the 3rd European Conference on Computer Vision, Stockholm, Sweden, pages 179-186. Springer-Verlag, 1994.

[KM96] T. Kim and J.P. Muller. Automated urban area building extraction from high resolution stereo imagery. Image and Vision Computing, 14:115-130, 1996.

[Kos93] A. Koschan. What is new in computational stereo since 1989: A survey on ereo papers. Technical report, Department of Computer Science, University of Berlin, august 1993.

[Mon87] O. Monga. An optimal region growing algorithm for image segmentation. International Journal of Pattern Recognition and Artificial Intelligence, 1(3):351-375, 1987.

[OC89] G.P. Otto and T.K. Chau. A region-growing algorithm for matching of terrain images. Image and Vision Computing, 7(2):83-94, 1989. 
[ZDFL94] Z. Zhang, R. Deriche, O.D. Faugeras, and Q.T. Luong. A robust technique for matching two uncalibrated images through the recovery of the unknown epipolar geometry. Artificial Intelligence, 78(1-2):87-119, 1994. Appeared in October 1995, also INRIA Research Report No.2273, May 1994.

INRIA 
Unit`e de recherche INRIA Lorraine, Technopôle de Nancy-Brabois, Campus scientifique, 615 rue du Jardin Botanique, BP 101, 54600 VILLERS LÈS NANCY

Unit'e de recherche INRIA Rennes, Irisa, Campus universitaire de Beaulieu, 35042 RENNES Cedex Unit'e de recherche INRIA Rhône-Alpes, 655, avenue de l'Europe, 38330 MONTBONNOT ST MARTIN Unit'e de recherche INRIA Rocquencourt, Domaine de Voluceau, Rocquencourt, BP 105, 78153 LE CHESNAY Cedex Unit ${ }^{\prime} e$ de recherche INRIA Sophia-Antipolis, 2004 route des Lucioles, BP 93, 06902 SOPHIA-ANTIPOLIS Cedex

Éditeur

INRIA, Domaine de Voluceau, Rocquencourt, BP 105, 78153 LE CHESNAY Cedex (France) http://www.inria.fr ISSN 0249-6399 